\title{
Tumor Vascularity and Lens Culinaris Agglutinin Reactive $\alpha$-Fetoprotein Are Predictors of Long-Term Prognosis in Patients with Hepatocellular Carcinoma after Percutaneous Ethanol Injection Therapy
}

\author{
HITOSHI FUKUDA \\ The Second Department of Medicine, Kurume University School of Medicine, \\ Kurume 830-0011, Japan
}

\begin{abstract}
Summary: Percutaneous ethanol injection therapy (PEIT) is now widely used for small hepatocellular carcinoma (HCC). However, only limited information is available regarding predictors of long-term prognosis of patients with small HCC after PEIT. The relationship of pretreatment clinicopathologic and biologic factors (age, sex, virus marker, Child-Pugh classification, tumor size, number of tumor, histologic grade, tumor staining, $\alpha$-fetoprotein level, AFP-L3\%, and Ki67 labelling index) to long-term prognosis in 41 patients with HCC were studied. Over-all survival rates of patients were $73.5 \%$ in 3-year, $34.7 \%$ in 5 -year, and $27.0 \%$ in 7 -year. Of the 12 variables investigated, Child-Pugh classification $(p=0.0243)$, histologic grade $(p=0.0098)$, tumor staining $(p=0.0012)$, AFP-L3\% (0.0093), and Ki67 labelling index $(p=0.0467)$ were significantly associated with the long-term prognosis by univariate analysis. According to the multivariate analysis using Cox's proportional hazard model with step-wise method, tumor staining $(p=0.0024)$ and AFP-L3\% $(p=0.0137)$ were significantly associated with the long-term prognosis after PEIT. In conclusion, the study found that positive tumor staining of $\mathrm{HCC}$ and positive AFP-L3\% in serum were the predictor of poor prognosis in patients with HCC after PEIT.
\end{abstract}

Key words hepatocellular carcinoma, tumor vascularity, AFP-L3, prognostic factor

\section{INTRODUCTION}

Percutaneous ethanol injection therapy (PEIT) for hepatocellular carcinoma (HCC) has gained a wide popularity because of its high antitumoral efficacy coupled with its relatively simple and inexpensive application [1-3]. Ethanol dehydrates and coagulates tumorous tissue on contact and causes vascular occlusion. As a result, complete ablation of cancerous nodules can be expected if the procedure is performed correctly. For the treatment of patients with small HCC, the high antitumoral efficacy and the absence of related mortality produce a survival rate that is almost the same as that of surgical patients. The optimal tumor size for PEIT is considered to be less than $3 \mathrm{~cm}$ in diameter and less than 3 nodules [4]. However, predictors of long-term prognosis of patients with small HCC, especially when they are treated by PEIT, have not been sufficiently clarified. Tumor size, number of tumors, and $\alpha$-fetoprotein (AFP) level are reported to be important prognostic factors for HCC treated by surgery [5]. Recently, histologic grade, tumor size, underlyning liver disease, tumor vascularity and regular follow-up after PEIT are reported to be important prognostic factors for small HCC treated by PEIT [6-8].

The biologic features that are indicative of the clinical aggressiveness of cancer cells and that are useful for identification of patients at low and high risk have been found to also aid in the prediction of treatment effects and patient survival. Such putative 
predictors of $\mathrm{HCC}$ in diagnosis and treatment include tumor markers $[9,10]$, pathological indices, proliferation indices [11], genetic alterations [12] and adhesion molecules [13]. AFP-L3, an isoform of AFP, is reactive with lens culinaris agglutinin and is known to be a highly specific and sensitive tumor marker of HCC [9]. Kuromatsu et al. [14] reported that the proportion of AFP-L3 levels was significantly higher in patients with moderately or poorly differentiated HCC than in those with welldifferentiated HCC. Yamashita et al. [5] reported that the proportion of AFP-L3 levels closely correlated with clinical aggressiveness of $\mathrm{HCC}$, and that a determination of AFP-L3 levels in serum permitted the stratification of patients with $\mathrm{HCC}$ according to the prognosis.

This study investigated the prognostic factors of patients with small HCC after PEIT using the reported clinicopathologic and biologic factors [5-7] including tumor vascularity [8], AFP-L3\% [10], and immunohistochemical staining of Ki67, which is a proliferation index of cancer cells [11].

\section{PATIENTS AND METHODS}

\section{Patients}

From April 1989 to December 1992, 252 patients with $\mathrm{HCC}$ less than $3 \mathrm{~cm}$ in diameter and three or fewer 3 nodules were treated by PEIT as their initial therapy in Kurume University Hospital. Of these, 41 patients whose serum was tested both for total AFP concentration and AFP-L3 level before treatment were selected as the subjects for this study. The diagnosis of HCC was histologically confirmed in all patients by tumor biopsy according to the classification of primary liver cancer [15] by the Liver Cancer Study Group of Japan. When more than one HCC nodule was present, the histologic grade of the largest tumor was applied for this evaluation.

\section{Measurement of total AFP concentrations and AFP- L3\%}

Total AFP concentrations in serum samples were determined by radio-immunoassay. The proportion of AFP-L3 was determined using the AFP Differentiation Kit L (Wako Pure Chemical Industries Ltd, Osaka, Japan). The proportion of AFP-L3 was calculated as (AFP-L3 Concentration/ Total AFP Concentration) $\times 100(\%)$. A cut-off level of $15 \%$ was used on the basis of previous study [9]. A positive AFP-L3 level was defined as an AFP-L3 level greater than the cut-off level.

\section{Evaluation of tumor vascularity}

Thirty-six patients received hepatic angiography before any treatment and biopsy. On angiogram, the presence of tumor stain was evaluated and tumors were classified as positively or negatively stained. Tumors in which the size of the tumor stain was smaller than the size of the tumor as estimated by ultrasonography, indicating heterogeneity of vascularity within the tumor, were defined as having positive staining. In 5 patients who did not receive hepatic angiography before treatment, tumor vascularity of their $\mathrm{HCC}$ were evaluated using dynamic enhanced-computed tomography (D-CT) as reported by Takayasu et al. [16]. In brief, tumors showing typical high density area on early phase of D-CT were classified as positive staining.

\section{Percutaneous ethanol injection therapy}

PEIT was performed under ultrasonographic guidance (SSD-650, Aloka, Tokyo) with $5 \mathrm{MHz}$ sector probe and guide device. In one treatment session, $2-5 \mathrm{ml}$ of $90 \%$ ethanol with local anesthetic was injected into the tumor. This injection was repeated twice a week for up to 6-10 sessions. The total volume of injected ethanol ranged from 15 to 50 $\mathrm{ml}$. The end point of treatment was determined according to the post treatment D-CT. In principle, the standard for the completion of the treatment was the presence of a necrotic low-density area in the site of the enhanced tumor on pretreatment D-CT and the undetectability of tumor enhancement on the early phase of D-CT. If the tumor had not been enhanced by pretreatment D-CT, the presence of a necrotic low density area larger than the area of portal perfusion which had been detected at the late phase of pretreatment D-CT was used to define as the endpoint of the therapy.

Follow-up after treatment and treatment of recurrent tumor (s)

The patients were followed-up regularly for the detection of recurrence by ultrasonography every 3 months and by D-CT every 6 months at outpatient clinics. When recurrence(s) was diagnosed by these diagnostic imagings or by additional tumor biopsies, patients were treated appropriately by PEIT as well as by such treatments as transcatheter arterial embolization [17] and regional chemotherapy [18] according to the extent of the recurrent tumor(s). The period of observation ended on December 28, 1996. 
TABLE 1.

Characteristics of patients with small hepatocellular carcinoma

\begin{tabular}{|c|c|c|c|}
\hline & \multicolumn{2}{|c|}{ proportion of AFP-L3 } & \multirow{2}{*}{$\mathrm{p}$ value } \\
\hline & positive $(\geq 15 \%)$ & negative $(<15 \%)$ & \\
\hline patients & 15 & 26 & \\
\hline \multicolumn{4}{|l|}{$\operatorname{sex}$} \\
\hline men/women & $9 / 6$ & $20 / 6$ & n.s. \\
\hline \multicolumn{4}{|l|}{ age } \\
\hline mean $\pm S D$ & $65.5 \pm 8.4$ & $61.7 \pm 6.8$ & n.s. \\
\hline \multicolumn{4}{|l|}{ HCV antibody } \\
\hline positive/negative & $14 / 1$ & $24 / 2$ & n.s. \\
\hline \multicolumn{4}{|l|}{ HBs antigen } \\
\hline positive/negative & $1 / 14$ & $2 / 24$ & n.s. \\
\hline \multicolumn{4}{|c|}{ Child-Pugh classification } \\
\hline $\mathrm{A} / \mathrm{B} / \mathrm{C}$ & $2 / 11 / 2$ & $16 / 9 / 1$ & n.s. \\
\hline \multicolumn{4}{|l|}{ tumor size } \\
\hline $\begin{array}{l}\text { tumor number } \\
\text { single/multiple } \\
\text { histological grade }\end{array}$ & $10 / 5$ & $15 / 11$ & n.s. \\
\hline $\begin{array}{c}\text { well-/mod.-poor. } \\
\alpha \text {-fetoprotein }(\mathrm{ng} / \mathrm{ml})\end{array}$ & $3 / 12$ & $14 / 12$ & $\mathrm{p}=0.0735$ \\
\hline $\begin{array}{c}\text { mean } \pm \mathrm{SD} \\
\text { tumor staining }\end{array}$ & $201.4 \pm 233.6$ & $96.3 \pm 124.8$ & n.s. \\
\hline positive/negative & $11 / 4$ & $13 / 13$ & n.s. \\
\hline Ki67 labelling index & $9.5 \pm 9.7$ & $6.0 \pm 7.1$ & n.s. \\
\hline
\end{tabular}

HCV antibody: hepatitic C antibody; HBs antigen: hepatitis B surface antigen

Evaluation of proliferative activity of HCC using Ki67 immunohistochemical staining

For immunohistochemical demonstration of Ki67 [11], the antibodies used were rabbit polyclonal antibody MIB-1 (IgG1, DAKO, Denmark) that recognizes native Ki67 antigen and recombinant fragments of the Ki67 molecule. Paraffin-embedded blocks of tumor samples were cut at $4 \mu \mathrm{m}$ thickness and put on 3-aminopropyl aminopropyltriethylxylane (APES)-coated slides. Paraffin sections were dewaxed in xylene and rehydrated in a series of graded alcohols according to histopathological standards. Deparaffined tissue sections were washed in distilled water, and immersed $10 \mathrm{mM}$ citrate buffer ( $\mathrm{pH} \mathrm{6.0)}$ ) at $120^{\circ} \mathrm{C}$ for $5 \mathrm{~min}$ in an autoclave [19]. The sections were allowed to cool down to room temperature (approximately $3 \mathrm{hs).} \mathrm{Next,} \mathrm{to}$ block the endogenous peroxidase activity, the slides were immersed in methanol with $0.3 \%$ hydrogen peroxide for $30 \mathrm{~min}$. The slides were rinsed twice in phosphate-buffered saline (PBS)-Tween20, and blocked with appropriate normal serum in PBS for $30 \mathrm{~min}$ to minimize non-specific absorption of the antibodies to the slides. The slides were incubated with the primary antibody for 15 to $18 \mathrm{hs}$ at $4^{\circ} \mathrm{C}$. The optimal dilution was 1:50 for Ki67 (MIB-1). After three further rinses in PBS-Tween20, the slides were incubated for $30 \mathrm{~min}$ with biotinylated secondary antibodies (ABC Vectastain Kit, Vector Laboratories, CA). They were subsequently incubated with streptotavidin-biotin peroxidase complex (ABC Vectastain Kit, Vector Laboratories, CA). Sections were washed twice for 5 min with PBS, developed with 3,3-diaminobenzine tetrahydrochroride (DAB) substrates (Sigma, UK), lightly counterstained with hematoxylin, dehydrated, cleared, and mounted.

For evaluation of immunostaining of Ki67, all labelled nuclei with brown-colored staining were regarded as a positive staining of the nuclei regardless of staining intensity or pattern. In each case, more than 500 cells were actually counted at a final magnification of $\times 400$ in each section to determine the labelling index (L.I.), which is the number (\%) of positive cells. As positive controls, specimens of normal tonsil, which had been proven to be positive for Ki67 (MIB-1) in more than $80 \%$ of 
cells, were applied. For negative controls, the antibody was replaced by equivalent amounts of normal rabbit IgG.

\section{Statistic analysis}

Data are expressed as mean $\pm \mathrm{SD}$ with the range. Chi-square test, Mann-Whitney U test, and Fisher's exact probability test were used appropriately to compare patients' background factors and tumor factors. To examine the variables as predictive factors of prognosis in patients with HCC after PEIT, 12 clinicopathologic and biologic variables were selected from the items mentioned above, and then stratified as listed in Table 2. Age was stratified according to the mean (63 years), Ki67 labelling index was stratified according to the median (4.4\%), ChildPugh classification, tumor size, number of tumor, histologic grade, and tumor staining were stratified according to the previous report [6,8], and AFP level and AFP-L3 levels (\%) were stratified as positive and negative according to the cut-off value. The survival rate of patients in relation to each of these variables was calculated by the Kaplan-Meier method. The estimated significance of the differences in the survival was analyzed by the log rank test. The significance of the prognostic value of the variables was estimated with Cox's multivariate proportional hazard model [20]. Differences were considered statistically significant when $\mathrm{p}<0.05$.

\section{RESULTS}

As shown in Table 1, the patients were 29 men and 12 women with a mean age of $63 \pm 7.5$ years (range, $48-80$ years). The etiology of liver disease was posthepatitis $\mathrm{C}$ in 38 patients, and posthepatitis $B$ in 3 patients. For patients who were admitted before 1991, stored serum was used for analysis of virus markers. Most patients had compensated liver disease; according to the Child-classification, 18 were grade A, 20 were grade $\mathrm{B}$ and 3 were grade $\mathrm{C}$. In regard to $\mathrm{HCC}$ tumors, the mean maximal tumor size was $17.8 \pm 6.1 \mathrm{~mm}$ (range, 7-30 mm). Twentyfive patients $(61.0 \%)$ had a uninodular tumor and 16 had a multinodular tumor. Histologic grade [15] of HCC was well-differentiated HCC in 17 patients $(41.5 \%)$ and moderately or poorly differentiated HCC in 24 patients. According to the evaluation of the tumor vascularity described in Methods, 24 patients $(58.5 \%)$ with $\mathrm{HCC}$ were classified having positive staining. The total mean AFP concentration of the 41 patients was $134.7 \pm 175.2 \mathrm{ng} / \mathrm{ml}$ (range, 4$868 \mathrm{ng} / \mathrm{ml}$ ), and the Ki67 labelling index was 7.3土 $8.3 \%$ (range, $0-38.8 \%$ ).

\section{Comparison of clinicopathologic and biologic factors in relation to positivity for AFP-L3\%}

Of the 41 patients, 15 were positive for AFPL3\% and the other 26 patients were negative. In relation to the positivity for AFP-L3\%, 11 clinicopathologic and biologic variables were examined (Table 1). The positive/negative for AFPL3\% was significantly related to the tumor size $(\mathrm{p}=0.023)$, and the histologic grade showed a somewhat weaker relationship $(\mathrm{p}=0.0735)$. There were no significant differences between these two

TABLE 2.

Categorization of variables

\begin{tabular}{llc}
\hline variables & \multicolumn{1}{c}{ categorization } & number of cases \\
\hline age & $\leq 62 / 63 \leq$ & $20 / 21$ \\
Sex & female/male & $12 / 29$ \\
HCV antibody & negative/positive & $3 / 38$ \\
HBs antigen & negative/positive & $38 / 3$ \\
Child-Pugh classification & grade A/grade B or C & $18 / 23$ \\
tumor size (mm) & $\leq 20 / 21$-30 & $27 / 14$ \\
number of tumor & uninodular/multinodular & $25 / 16$ \\
histological grade & well-/moderately or poorly & $17 / 24$ \\
$\alpha$-fetoprotein (ng/ml) & $\leq 19 / 20 \leq$ & $12 / 29$ \\
AFP-L3\% & negative/positive & $26 / 15$ \\
Ki67 labelling index & $\leq 4,4 \% / 4.5 \% \leq$ & $23 / 18$ \\
tumor staining & negative/positive & $17 / 24$
\end{tabular}

HCV antibody: hepatitic C antibody; HBs antigen: hepatitis B surface antigen; well-: well-differentiated hepatocellular carcinoma; moderately or poorly: moderately and poorly differentiated hepatocellular carcinoma 
TABLE 3.

Cumulative survival rate of patients after PEIT stratified by variable using Kaplan-Meier method

\begin{tabular}{|c|c|c|c|c|}
\hline \multirow{2}{*}{ Variables } & \multicolumn{3}{|c|}{ estimated survival (\%) } & \multirow{2}{*}{$\mathrm{p}$ value } \\
\hline & 3-year & 5-year & 7-year & \\
\hline Child-Pugh classification & & & & $\mathrm{p}=0.0243$ \\
\hline A & 88.9 & 42.1 & 42.1 & \\
\hline $\mathrm{B}$ and $\mathrm{C}$ & 60.9 & 22.4 & 16.8 & \\
\hline histological grade & & & & $\mathrm{p}=0.0089$ \\
\hline well-diff. HCC & 81.9 & 52.0 & 52.0 & \\
\hline mod. and poorly diff. HCC & 58.3 & 7.5 & - & \\
\hline AFP-L3\% & & & & $\mathrm{p}=0.0093$ \\
\hline less than $14 \%$ & 80.6 & 38.6 & 38.6 & \\
\hline more than $15 \%$ & 53.3 & 8.3 & - & \\
\hline Ki67 labelling index & & & & $\mathrm{p}=0.0467$ \\
\hline less than $4.4 \%$ & 77.8 & 46.1 & 35.8 & \\
\hline more than $4.5 \%$ & 55.6 & 13.1 & - & \\
\hline tumor staining & & & & $\mathrm{p}=0.0012$ \\
\hline negative & 88.2 & 66.6 & 47.6 & \\
\hline positive & 62.2 & 11.4 & - & \\
\hline total cases & 73.5 & 34.7 & 27.0 & - \\
\hline
\end{tabular}

TABLE 4.

Significant risk factors for survival after PEIT; multivariate analysis tested by Cox's proportional hazard model

\begin{tabular}{ccccc}
\hline Variables & Beta & Standard error & Chi-square & p value \\
\hline $\begin{array}{c}\text { tumor staining } \\
\text { positive/negative }\end{array}$ & -1.440 & 0.475 & 9.204 & 0.0024 \\
$\begin{array}{c}\text { AFP-L3\% } \\
\text { positive/negative }\end{array}$ & -1.050 & 0.426 & 6.081 & 0.0137 \\
\hline
\end{tabular}

groups in other variables.

Survival rate in patients with HCC after PEIT and the results of univariate analysis

At the time of analysis, 14 patients were alive and 27 patients had died. The estimated probability of survival for all of the 41 patients, calculated by means of the Kaplan-Meier method, was $73.5 \%$ at 3 years, $34.7 \%$ at 5 years, and $27.0 \%$ at 7 years. The differences in survivals of patients with HCC after PEIT among the various subgroups identified with each prognostic factor were evaluated using log rank tests. Child-Pugh classification $(\mathrm{p}=0.0243)$, histologic grade $(\mathrm{p}=0.0089)$, AFP-L3\% $(\mathrm{p}=0.0093), \mathrm{Ki67}$ labelling index $(\mathrm{p}=0.0467)$, and tumor staining $(p=0.0012)$ were found to be significantly related to longer survival of the patients with HCC after PEIT. Three-, 5-, and 7-year survival rates for these significant groups are listed in Table 3. The other 7 factors showed no relation to the survival rate.

\section{The results of multivariate analysis}

Multivariate regression analysis was conducted to assess the independent prognostic importance of each variable studied using Cox's proportional hazard model. As listed in Table 4, tumor staining and AFP-L3\% were found to be significant independent prognostic factors related to the patients' survival after PEIT. The same results were obtained with forward and backward analysis.

\section{DISCUSSION}

Because the therapeutic efficacy of PEIT in terms of percentage of necrosis is high, the overall 5-year survival rate of patients after PEIT [6] is almost the 
same as that of surgical patients [5]. Recently, several studies have reported on prognostic factors of long-term survival of patients [6-8] and predictive factors of recurrence [21,22] after PEIT. This study was designed to investigate the two novel prognostic factors AFP-L3\% [10], and immunohistochemical staining of Ki67 [11], along with the previously reported prognostic factors [6-8] in patients with small HCC after PEIT.

According to the result of the current study, tumor vascularity was found to be an independent prognostic factor related to the patients' survival after PEIT by the multivariate regression analysis using the stepwise method. Toyoda et al. [8] first reported the importance of tumor vascularity in predicting the long-term prognosis of patients with small HCC treated by PEIT. They observed that the vascularity of small HCCs and their histologic grade were statistically significant, and that the survival rate after PEIT in patients with HCC showing negative tumor staining was statistically better than that of those with HCC showing positive tumor staining. In this study, we also observed that the vascularity (negative/positive) of the HCCs studied was significantly $(\mathrm{p}=0.011$ by chi-square test, data was not shown) related to the histologic grade (well/moderately and poorly differentiated HCC), and that the difference in the long-term survival rates of these two groups was statistically significant (Table 3 ). In patients with small HCC after PEIT, Tanaka et al. [6] reported that histologic grade was the most important prognostic factor of the long-term survival. These results indicated that the tumor vascularity was the most significant prognostic factor of the long-term survival in patients with small HCC after PEIT.

AFP-L3\% was found to be another significant and independent prognostic factor related to the patients' survival after PEIT by multivariate regression analysis using the stepwise method. AFP-L3\% in patients with HCC was reported to be significantly elevated in patients with moderately or poorly differentiated HCC as compared to in those with well-differentiated HCC [9,14]. In this study, however, the relationship between AFP-L3\% and histologic grade was not significant $(p=0.0735)$. The number of patients in this study was small, and this might be one reason to no significant difference was observed. Yamashita et al. [10] reported that the positivity of AFP-L3\% was a significant marker of poor prognosis for $\mathrm{HCC}$, and that multiple recurrences of $\mathrm{HCC}$ and portal vein tumor thrombus were observed significantly more often in patients with positive AFP-L3 than in those with negative AFP-L3 status. These results also indicated that AFP-L3 status was a significant prognostic factor of the long-term survival in patients with small HCC after PEIT.

The significance of immunohistochemical staining of Ki67, which is a proliferation index of cancer cells [11], was also studied. Yoshimoto et al. [11] reported that Ki67 labelling index of HCC cells was significantly higher in those who had multiple recurrence after hepatectomy than in those who had single-lesion recurrence, and that the cumulative survival rate was significantly lower in patients with higher Ki67 labelling indexes than in those with lower ones. In this study, the pathologic factors such as Ki67 labelling index and histologic grade were significant predictors of long-term prognosis in patients with small HCC after PEIT by univariate analysis. However, these factors were not significant under the multivariate analysis. The specimens studied were obtained by fine-needle biopsy, rather than by surgical resection. These pathologic factors such as Ki67 labelling index and histologic grade were evaluated from biopsied specimens, which might represent the features of only a localized part of the whole tumor. Therefore there could be over- or underestimation of the pathologic features in some HCC specimens evaluated from fine-needle biopsy.

The main point of interest of this study is that the results permit stratification of $\mathrm{HCC}$ patients who are candidates for PEIT according to their prognosis. Therefore, the results of this study may be helpful in determining treatment strategies. Patients with poor prognostic factors may require more aggressive therapy, such as PEIT combined with transcatheter arterial embolization [23], microwave coagulation therapy, and hepatic resection.

ACKNOWLEDGMENTS: The author wishes to thank Professor Kyuichi Tanikawa and Dr. Masatoshi Tanaka.

\section{REFERENCES}

1. Livraghi T, Salmi A, Bolondi L, Martin G, Arienti V et al. Small hepatocellular carcinoma: percutaneous ethanol injection-result in 23 patients. Radiology 1988; 168:313.

2. Shiina S, Tagawa K, Unuma T, Fujino H, Uta $\mathrm{Y}$ et al. Percutaneous ethanol injection therapy of hepatocellular carcinoma: analysis of 77 patients. AJR 1990; 155:12211226.

3. Livraghi T, Giorgio A, Marin G, Salmi A, Sio I et al. Hepatocellular carcinoma and cirrhosis in 746 patients: long-term results of percutaneous ethanol injection. Radiology 1995; 197:101-108. 
4. Ehara M, Ohto M, Sugiura N, Kita K, Yoshikawa M et al. Percutaneous ethanol injection for the treatment of small hepatocellular carcinoma: study of 95 patients. J Gastroenterol Hepatol 1990; 5:616-626.

5. The liver Cancer Study Group of Japan. Predictive factors for long term prognosis after partial hepatectomy for patients with hepatocellular carcinoma in Japan. Cancer 1994; 74:2772-2780.

6. Tanaka M, Shimada M, Ono N, Tateishi Y, Shimauchi Y et al. Prognosis of patients with small hepatoma after percutaneous injection of ethanol. In: Progress in Hepatology, vol 3, ed. Yamanaka M, Toda G and Tanaka T, Elsevier, Amsterdam, pp69-77, 1997.

7. Lencioni R, Bartolozzi C, Caramella D, Paolicchi A, Carrai $M$ et al. Treatment of small hepatocellular carcinoma with percutaneous ethanol injection. Cancer 1995; 76:1737-1746.

8. Toyoda H, Kumada T, Nakano S, Takeda I, Sugiyama K et al. Significance of tumor vascularity as a predictor of long-term prognosis in patients with small hepatocellular carcinoma treated by percutaneous ethanol injection therapy. J Hepatology 1997; 26:1055-1062.

9. Taketa K, Endo Y, Sekiya C, Tanikawa K, Koji T et al. A collaborative study for the evaluation of lectin-reactive alpha-fetoproteins in early detection of hepatocellular carcinoma. Cancer Res 1993; 53:5419-5423.

10. Yamashita F, Tanaka M, Satomura S, and Tanikawa K. Prognostic significance of Lens culinaris agglutinin Areactive alpha-fetoprotein in small hepatocellular carcinomas. Gastroenterology 1996; 111:996-1001.

11. Yoshimoto J, Iwata T, Takamori, Kojima K, and Futagawa S. Usefulness of monoclonal antibody Ki-67 as a prognostic factor of hepatocellular carcinoma. Int Hepatol Communications 1997; 6:209-218.

12. Oda T, Tsuda H, Scarpa A, Sakamoto M, and Hirohashi S. p53 mutation spectrum in hepatocellular carcinoma. Cancer Res 1992; 52:6358-6364.

13. Ihara A, Koizumi H, Hashizume R, and Uchikoshi $T$. Expression of epithelial cadherin and $\alpha$-and $\beta$-catenins in nontumoral livers and hepatocellular carcinomas.
Hepatology 1996; 23:1441-1447.

14. Kuromatu R, Tanaka M, and Tanikawa K. Serum alphafetoprotein and lens culinaris agglutinin-reactive fraction of alpha-fetoprotein in patients with Hepatocellular Carcinoma. Liver 1993; 13:177-182.

15. Liver Cancer Study Group of Japan. Classification of primary liver cancer. Kanehara \& Co., Tokyo, 1997.

16. Takayasu K, Furukawa H, Wakao F, Muramatsu Y, Abe $\mathrm{H}$ et al. CT diagnosis of early hepatocellular carcinoma: sensitivity, findings, and CT-pathologic correlation. AJR 1995; 164:885-890.

17. Yamada R, Sato M, Kawabata M, Nakatsuka $H$, Nakamura K et al. Hepatic artery embolization in 120 patients with unresectable hepatoma. Radiology 1983; 148:397-401.

18. Ando E, Yamashita F, Tanaka M, and Tanikawa K. A novel chemotherapy for advanced hepatocellular carcinoma with tumor thrombosis of the main trunk of the portal vein. Cancer 1997; 79:1890-1896.

19. Shin R-W, Iwaki T, Kitamoto T, and Tateishi J. Hydrated autoclave pretreatment enhances TAU immunoreactivity in formalin-fixed normal and Alzheimer's disease brain tissues. Laboratory Investigation 1991; 64:693-702.

20. Christensen E. Multivariate survival analysis using Cox's regression model. Hepatology 1987; 7:1346-1358

21. Ishii H, Okada S, Nose H, Okusaka T, Yoshimori M et al. Local recurrence of hepatocellular carcinoma after percutaneous ethanol injection. Cancer 1996; 77:17921796.

22. Pompili M, Rapaccini GL, Luca F, Caturelli E, Astone A et al. Risk factors for intrahepatic recurrence of hepatocellular carcinoma in cirrhotic patients treated by percutaneous ethanol injection. Cancer 1996; 79:15011508.

23. Tanaka K, Nakamura S, Numata K, Okazaki H, Endo O et al. Hepatocellular carcinoma: treatment with percutaneous ethanol injection and transcatheter arterial embolization. Radiology 1992; 185:457-460. 